\title{
The gene expression level of enzymatic and non-enzymatic antioxidant system of potato plants under chloride salinity
}

\author{
Murgan O.K.*, Efimova M.V. \\ Tomsk State University, Tomsk, Russia \\ *e-mail: reborn_rinni@mail.ru
}

The growing season duration for potato plants is one of the key criteria for their regionalization in a certain climate zone. For the Siberian region, characterized by a short summer season, early-maturing varieties are most preferred. However, the increase of saline areas sets priority on salt tolerance varieties. Salinization is negatively affects the implementation of many physiological processes in plants, largely due to the generation of reactive oxygen species and the development of oxidative stress, which leads to disruption of the photosynthetic apparatus, a decrease in the intensity of photosynthesis and, as a consequence, a decrease in the productivity of plants; induction of aging or premature death of the plant. In response to the oxidative stress progress and metabolic disturbances, the plant responds by activating the cellular antioxidant system, which includes antioxidant enzymes (catalases, peroxidases, superoxide dismutases, etc.) and low-molecular-weight organic compounds with antioxidant properties (proline, phenolic compounds, carotenoids). The gene expression of antioxidant system will compare the functioning of the protective system for two varieties (early and mid-season) the plant Solanum tuberosum in response to chloride salinity. We estimated the effect of salinity on growth (length of axial organs, area of assimilating surface) and physiological (osmotic potential of cell exudate, lipid peroxidation degree, content of photosynthetic pigments, flavonoids, proline, activity of antioxidant enzymes) for early and mid-season varieties Solanum tuberosum (Zhukovsky early and Lugovskoy). The gene expression levels of enzymatic (APX1, APX3) and non-enzymatic (P5CS1, P5CR, PDH) plant protection were detected by real time PCR. For the first time, the causes of potato plant resistance to $\mathrm{NaCl}$ action for analyzed varieties were identified and a detailed comparison of the functioning of the protective systems of potato plants depending on the maturation period was made.

Acknowledgements: This study was supported by the Russian Science Foundation. 